\title{
Modelo de Dispersión Axial para Sistemas de Flujo Continuo Ajustado a las Condiciones de Borde
}

\author{
Jhonny I. Pérez ${ }^{(1)}$, Gerardo J. Aldana ${ }^{(2)}$ y Gabriel J. Arguello(3) \\ (1) Grupo de Investigación GISA, Facultad de Ingeniería, Univ. de La Guajira, Km 5 Vía a Maicao, \\ Riohacha-Colombia. (e-mail: jpemon@uniguajira.edu.co) \\ (2) Centro de Investigaciones del Agua (CIA), Facultad de Ingeniería, Univ. del Zulia, Av. 16 (Guajira) con \\ Av. Cecilio Acosta. Núcleo Técnico, Maracaibo-Venezuela. (e-mail: gerardoaldana_21@hotmail.com) \\ (3) Depto. de Matemática, Facultad de Ingeniería, Universidad del Zulia, Univ. del Zulia, Av. 16 (Guajira) \\ con Av. Cecilio Acosta. Núcleo Técnico, Maracaibo-Venezuela. (e-mail: garguello55@gmail.com)
}

Recibido Jun. 11, 2015; Aceptado Ago. 19, 2015; Versión final Oct. 8, 2015, Publicado Feb. 2016

\section{Resumen}

Se desarrolló un modelo matemático a partir de la ecuación general de dispersión y se comparó con tres modelos tradicionales. Para obtener los datos experimentales se realizaron ensayos hidráulicos utilizando litio como trazador agregado de forma puntual en el influente de un Reactor Anaerobio de Flujo Ascendente de 518 litros, alimentado con agua residual de la ciudad de Maracaibo-Venezuela a tiempos de retención hidráulico teórico de 10, 8, 5 y 4 horas. Se tomó muestras en el efluente y al final del lecho y manto de lodo. Los mejores ajustes se lograron con el modelo propuesto con desviación media absoluta entre 5 y $33 \%$ y desviación media relativa entre $-1,7$ y $-18 \%$. En todos los casos, el número de dispersión fue mayor a 0.01 indicando que no existió flujo pistón en el reactor.

\section{Axial Dispersion Model for Continuous Flow Systems Adjusted Boundary Conditions}

\begin{abstract}
In this study a mathematical model was developed from a general dispersion equation, which was compared with three traditional models. Tracer tests were carried injected lithium as a pulse at the reactor influent. Several samples were collected in an Upflow Anaerobic Sludge Bed reactor o 518 liters to obtain the experimental data. The reactor was feeding from municipal wastewater of Maracaibo city, Venezuela. Several theoretical retention times were evaluated between 4, 5, 8 and 10 hours. Samples were taken at the final effluent and at the end of the sludge blanket and bed level. Best fits were obtained with the proposed model giving absolute mean deviation between 5.0 and $33 \%$ and relative mean deviation between -1.7 and $-18 \%$. In most of the studies the dispersion number was lower than 0.01 concluding that plug flow did not exist in the reactor.
\end{abstract}

Keywords: axial dispersion; model; tracer; instantaneous injection; upflow reactor 


\section{INTRODUCCIÓN}

Ha sido tradicional el uso los modelos unidimensionales propuesto por Levenspiel (Levenspiel, 1999) para determinar la dispersión y con ella interpretar la distribución de los tiempos de residencia y los patrones de flujo en sistemas hidráulicos, con mucha aplicación en el área de la ingeniería sanitaria, ingeniería ambiental y ciencias afines, normalmente su aplicación se hace desde dos condiciones extremas: flujo pistón, para pequeña dispersión (PD), asignando un número de dispersión $\mathrm{d}<0,01$ e infinito para dispersiones grande conocido como mezcla completa (Lara et al., 2000; Arroyave et al., 2005, De Carvalho, 2008). Para el caso de mezcla completa o gran dispersión se usan las condiciones de recipiente abierto (GDra) y recipiente cerrado (GDrc). En este grupo de modelos tradicionales, se incluye el de tanques en serie (TS) (Martin, 2000).

La condición de recipiente abierto se logra cuando no existe alteración del flujo en los contornos, también llamado borde o frontera (entrada y salida), para la cual se publicó en los años 50 una solución analítica (Levenspiel y Smith, 1957). Las condiciones de recipiente cerrado se logran cuando existe perturbación del flujo en el contorno (Levenspiel, 1999), esta situación es bastante aproximada en muchos reactores reales e incluso en aquellos que presentan baja dispersión (Martin, 2000). Se afirmó que no existe solución analítica para estas condicione de borde y solo se presentan ecuaciones para estimar la dispersión a través de la media y la varianza (Levenspiel 1999). Por otro lado, un análisis de las condiciones de borde fue realizado para reactores de flujo continuo pero las fronteras solo se consideraron en términos de longitud finita e infinita (Wehner y Wilhelm, 1956), no contemplando la condición inicial y de borde de acuerdo a la inyección y recolección del trazador. Desde los años 70 se dio un importante avance que explica las condiciones de borde en reactores considerando el punto de inyección y recolección del trazador, aunque en este aspecto solo se reporte una ecuación adimensional para flujo pistón (Wen y Fan, 1975).

El modelo de TS describe el flujo considerando una cadena hipotética de reactores de tanques continuos agitados, cuando el número de tanques $(\mathrm{N})$ es igual 1 representa dispersión infinita (Martin, 2000). Este modelo tiene el inconveniente que $\mathrm{N}$ debería ser entero, además el valor de $\mathrm{N}$ ha sido relacionado con el número de Peclet $\left(\mathrm{P}_{\mathrm{e}}\right)$ o número de dispersión (d) a través de la varianza, esto equivale a suponer que el modelo de tanques en serie y recipiente cerrado son equivalentes; pero se ha observado gráficamente que un valor de $\mathrm{N}=1$ difiere significativamente de $\mathrm{N}=2$ (Martin, 2000). Por otro lado, considerando que el modelo TS es igual al modelo GDrc, se desarrolló una ecuación siguiendo el proceso de una reacción arbitraria y expandiendo la ecuación de dispersión en la serie de Taylor (Elgeti, 1996), pero según el análisis gráfico para una varianza equivalente a $\mathrm{N}=2$ y $\mathrm{d}=0,391$, las DTR son diferentes y el modelo GDrc exhibe un mayor pico que el modelo TS, esto significa que los dos modelos son de tendencia similar pero no iguales (Martin, 2000).

Una ecuación empírica fue propuesta para determinar el número de dispersión en lagunas de estabilización en función de sus dimensiones, tiempo de retención hidráulico y la viscosidad cinemática sin considerar las condiciones de borde (Polprasert y Bhattarai, 1985). Todos estos modelos (cerrado, abierto, tanque en serie) son adimensionales en su naturaleza y consecuentemente no describen el comportamiento del flujo dentro del reactor. Históricamente la interpretación de la distribución de los tiempos de residencia DTR se ha dado de manera superficial, frecuentemente esta interpretación ha sido limitada a determinar la media y la varianza de los datos sin considerar las bondades de ajuste de los modelos. Cuando la dispersión es grande la media es mucho mayor que 1, lo que demuestra su sensibilidad originando diferencias en la estimación (Devore, 2001; Martin, 2000).

Los sistemas hidráulicos usados para tratar las aguas residuales buscan mejorar la eficiencia cada día hasta poder reutilizarla (Passarini et al., 2012), la dispersión es un factor importante en este aspecto debido a la necesidad de tener un contacto íntimo entre los microorganismos y el sustrato. Dentro de estos sistemas se encuentran los RAFA conocido como UASB por sus siglas en inglés (Upflow Anaerobic Sludge Blanket) que es un reactor multifase (líquido-sólido-gas) y por consiguiente, el comportamiento global de la dispersión es el resultado de interacciones físicas interdependientes, propiedades del flujo, segregación de partículas y advección (Peña et al., 2006). El comportamiento hidrodinámico de reactores de flujo continuo puede evaluarse siguiendo el método experimental estímulo-respuesta, inyectando un trazador inerte de concentración conocida en el afluente (Rocha et al., 2000).

Por las consideraciones anteriores, se hizo necesario desarrollar la ecuación general de dispersión considerando las condiciones iniciales y de borde de acuerdo a la inyección y recolección del trazador, aplicado, que en nuestro diseño experimental se usó la inyección puntal (delta de Dirac) en el afluente con recolección en el efluente y en un punto intermedio del reactor, de esta manera la ecuación permite determinar la dispersión del flujo como parámetro de ajuste del modelo, lo que brinda confiabilidad en el resultado. 


\section{MATERIALES Y MÉTODOS}

La investigación se llevó a cabo en un RAFA (UASB) de 518 L (88,4 cm de ancho; 118,4 cm de largo y 55 $\mathrm{cm}$ de alto), diseñado como un modelo físico de un prototipo existente en Ginebra, Valle del CaucaColombia (Pérez y Aldana, 2013) construido en plástico transparente de $8 \mathrm{~mm}$. El montaje del experimento se realizó en las instalaciones del Centro de Investigaciones del Agua (CIA) de la Universidad del ZuliaVenezuela. Para su arranque se inocularon 105 litros de lodo (20\% v/v), $62 \mathrm{~L}$ proveniente de una cervecería local y $43 \mathrm{~L}$ de una laguna facultativa. Para control y funcionamiento del reactor, se midieron los siguientes parámetros físico-químicos: Temperatura, $\mathrm{pH}$, alcalinidad, demanda química de oxígeno (DQO), producción de biogás, contenido de metano, de acuerdo a lo establecido en el estándar métodos (APHA et al., 2005).

Los ensayos hidráulicos se realizaron a tiempos de to de: 10, 8, 5 y 4 horas, con 3 entradas alineadas en el fondo del reactor, luego se modificó el sistema para funcionar a to de 5 horas con $5,7,9,11,13$ y 15 entradas. Se inyectaron de forma instantánea $250 \mathrm{ml}$ de una solución de $\mathrm{LiCl}$ con una concentración de $5000 \mathrm{mgLi}^{+} / \mathrm{L}\left(\mathrm{C}_{0}=2,5 \mathrm{mg} / \mathrm{L}\right)$, con la ayuda de una bomba peristáltica (marca Cole Parmer modelo 7553-80 de 1-100 rpm) e inmediatamente se inició la recolección en el efluente (Inyección y recolección en flujo-IRF) y al final del lecho y manto de lodo (LML) que corresponde a inyección en flujo y recolección en el fluido residente (IFRFR) (Ver figura 1). El $\mathrm{Li}^{+}$fue seleccionado en ensayos a escala de laboratorio como mejor trazador comparando con $\mathrm{Cl}^{-}$y la rodamina WT (Perez et al., 2010). La recolección del trazador se hizo por un periodo mínimo de 4to, siendo recomendada una duración mínima de 3to (CEPIS, 2004). Las mediciones de $\mathrm{Li}^{+}$se realizaron en un espectrofotómetro de absorción atómica (marca Perkin Elmer, modelo 3110, método de llama gas aire-acetileno a 670,80 nm) con un límite de detección mínimo de 0,01 mg/L. Las muestras fueron preservadas con ácido nítrico, mantenidas en refrigeración y digestadas antes de su medición (APHA et al., 2005).

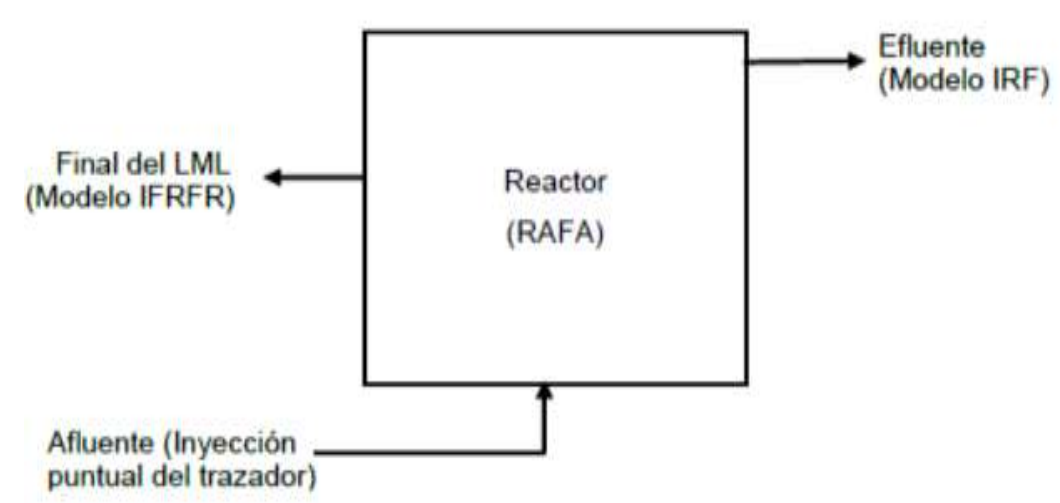

Fig. 1: Puntos de inyección y recolección del trazador que ilustran las condiciones iniciales y de borde para el desarrollo del modelo matemático

Los datos experimentales de la curva de DTR fueron transformados de forma adimensional para poder compararlos con los modelos tradicionales de pequeña dispersión (PD), GDra y TS (Levenspiel, 1999) de acuerdo a las ecuaciones 1,2 y 3 respectivamente y el modelo propuesto.

$\mathrm{E}_{\theta}=\frac{1}{2 \sqrt{\pi d}} \mathrm{e}^{\left[-\frac{(1-\theta)^{2}}{4 \mathrm{~d}}\right]}$

$\mathrm{E}_{\theta}=\frac{1}{2 \sqrt{\pi \theta d}} \mathrm{e}^{\left[-\frac{(1-\theta)^{2}}{4 \theta d}\right]}$

$E_{\theta}=\frac{N(N \theta)^{N-1}}{(N-1) !} e^{-N \theta}$

Donde: $\mathrm{E}_{\theta}=$ función de distribución de salida del trazador (adimensional), $\theta=$ tiempo de toma de muestra adimensional, $\mathrm{e}=$ base del logaritmo natural $=2,718 \ldots$ (adimensional), $\mathrm{N}=$ número de tanques en serie (adimensional). Para el desarrollo del modelo se aplicó transformada de Laplace a la ecuación general de dispersión (Holley, 1969, Kreft y Zuber, 1978) considerando la condición inicial (señal de pulso o instantánea) y dos condiciones de borde de acuerdo al punto de recolección del trazador. 


\section{DESARROLLO DEL MODELO DE DISPERSIÓN}

Retomando el planteamiento presentado en la metodología, se presenta el desarrollo del modelo de dispersión para una inyección tipo pulso o instantánea y dos puntos de recolección del trazador.

Solución de la ecuación de dispersión: Inyección y recolección en flujo (IRF)

Ecuación en Derivadas Parciales EDP: $\frac{\partial \mathrm{C}}{\partial \mathrm{t}}=\mathrm{D} \frac{\partial^{2} \mathrm{C}}{\partial \mathrm{x}^{2}}-\mathrm{v} \frac{\partial \mathrm{C}}{\partial \mathrm{x}}$ con dominio: $\left\{\begin{array}{c}\mathrm{t}>0 \\ 0<\mathrm{x}<\mathrm{H}\end{array}\right\}$

Condición inicial: $\mathrm{C}(\mathrm{x}, 0)=0$ para $0 \leq \mathrm{x} \leq \mathrm{H} \quad$ Condiciones de borde $\left\{\begin{array}{c}\mathrm{C}(0, \mathrm{t})=\frac{\mathrm{M}}{\mathrm{Q}} \delta(\mathrm{t}) \\ \lim _{\mathrm{x} \rightarrow+\infty} \mathrm{C}(\mathrm{x}, \mathrm{t})=0\end{array}\right\}$ para $\mathrm{t} \geq 0$

Donde: $\mathrm{C}=$ concentración del trazador $(\mathrm{mg} / \mathrm{L})$ en el tiempo $\mathrm{t}, \mathrm{x}=$ longitud $\mathrm{o}$ altura entre el punto de inyección y recolección del trazador $(\mathrm{m}), \mathrm{t}=$ tiempo transcurrido después de la inyección $(\mathrm{min}), \mathrm{D}=$ coeficiente de dispersión axial $\left(\mathrm{m}^{2} / \mathrm{min}\right), \mathrm{M}=$ masa del trazador inyectada $(\mathrm{mg}), \mathrm{Q}=$ caudal entrante al reactor, $\mathrm{H}=$ altura total del reactor, $\delta(\mathrm{t})=$ función delta de Dirac, $v=$ velocidad ascendente del fluido $(\mathrm{m} / \mathrm{min})$

Aplicando transformada de Laplace a ambos lados de la EDP del problema de borde de la ecuación 4 tenemos:

$\mathcal{L}\left\{\frac{\partial \mathrm{C}}{\partial \mathrm{t}}\right\}=\mathcal{L}\left\{\mathrm{D} \frac{\partial^{2} \mathrm{C}}{\partial \mathrm{x}^{2}}-\mathrm{v} \frac{\partial \mathrm{C}}{\partial \mathrm{x}}\right\}$

Aplicando las propiedades se obtiene: $s \bar{C}-C(x, 0)=D \frac{\partial^{2} \bar{C}}{\partial x^{2}}-v \frac{\partial \bar{C}}{\partial x}$,

Donde $\mathcal{L}\{\mathrm{C}(\mathrm{x}, \mathrm{t})\}=\overline{\mathrm{C}}(\mathrm{x}, \mathrm{s})$; Sustituyendo la condición inicial, $\mathrm{C}(\mathrm{x}, 0)=0$, y reordenando términos se obtiene:

$\mathrm{D} \frac{\partial^{2} \overline{\mathrm{C}}}{\partial \mathrm{x}^{2}}-\mathrm{v} \frac{\partial \overline{\mathrm{C}}}{\partial \mathrm{x}}-\mathrm{s} \overline{\mathrm{C}}=0$

Aplicando transformada de Laplace sobre la primera condición de borde se obtiene: $\mathcal{L}\{\mathrm{C}(0, \mathrm{t})\}=\mathcal{L}\left\{\frac{\mathrm{M}}{\mathrm{Q}} \delta(\mathrm{t})\right\}$

Como $M$ y $Q$ son constantes y $\mathcal{L}\{\delta(t)\}=1$, la condición transformada queda como:

$\overline{\mathrm{C}}(0, \mathrm{~s})=\frac{\mathrm{M}}{\mathrm{Q}}$

Considerando a s como si fuera un parámetro "temporalmente", la ecuación diferencial parcial 5 se puede reescribir como una ecuación diferencial ordinaria (EDO). La condición 6 se puede reescribir con respecto a $\mathrm{x}$, esto permite plantear el problema de contorno con EDO dado por la ecuación 7 .

EDO: $\mathrm{D} \frac{\mathrm{d}^{2} \overline{\mathrm{C}}}{\mathrm{dx}^{2}}-\mathrm{v} \frac{\mathrm{d} \overline{\mathrm{C}}}{\mathrm{dx}}-\mathrm{s} \overline{\mathrm{C}}=0$

Condiciones de borde: $\left\{\begin{array}{c}\bar{C}(0)=\frac{M}{Q} \\ \lim _{x \rightarrow+\infty} \bar{C}(x)=0\end{array}\right\}$

La ecuación característica asociada a la EDO del problema 7 es: $\mathrm{Dm}^{2}-\mathrm{vm}-\mathrm{s}=0$, Resolviendo la ecuación característica, aplicando las condiciones de borde, y resolviendo en el plano de las transformadas s de Laplace, se obtiene la siguiente ecuación (ya presentada por: Kreft y Zuber, 1978) 
$\mathrm{C}(\mathrm{x}, \mathrm{t})=\frac{\mathrm{M}}{\mathrm{Q}} \frac{\mathrm{x}}{\sqrt{4 \pi \mathrm{Dt}^{3}}} \mathrm{e}\left[-\frac{(\mathrm{x}-\mathrm{vt})^{2}}{4 \mathrm{Dt}}\right]$

La ecuación 8 fue rescrita y usada para interpretar curvas de DTR en sistemas de flujo continuo, tal como se muestra en la ecuación 9 (Werner et al., 1997)

$C(x, t)=\frac{M}{V} \frac{1}{\sqrt{4 \pi \frac{D}{v x}\left(\frac{t}{\bar{t}}\right)^{3}}} e\left[-\frac{\left(1-\frac{t}{t_{o}}\right)^{2}}{4 \frac{D t}{v x \bar{t}}}\right]$

Donde: $\mathrm{V}=$ volumen del reactor $(\mathrm{L}), \overline{\mathrm{t}}=$ tempo de retención hidráulico experimental $(\mathrm{min})$.

Recordando que $d=D / v x$ y $\theta=t / \bar{t}=$ tiempo de retención hidráulico adimensional, la ecuación 9 es similar a la presentada para una inyección instantánea sin publicar su desarrollo ni las condiciones de borde (Levenspiel y Smith, 1957). Luego se especificó que aplica para gran dispersión con recipiente abierto, lo que es adecuado debido a que no hay perturbación del flujo (Levenspiel, 1972), pero con una diferentica en el valor de $\theta$ del radical, el cual fue presentado de forma lineal (ver ecuación 2), debiendo ser $\theta^{3}$. En 1999, la ecuación 9 fue presentada omitiendo el valor de $\theta$ en el radical (Levenspiel, 1999), la cual fue usada para ajustar datos experimentales de la DTR en RAFA (De Carvalho, 2006; Gupta y Bansal, 2010).

Solución de la ecuación de dispersión: inyección en el flujo y recolección en el fluido residente (IFRFR). Tomando la ecuación 4, se definen la nueva condición de borde como se muestra e la ecuación 10.

Condiciones de borde: $\left\{\begin{array}{c}\mathrm{C}(0, \mathrm{t})=-\left.\frac{\mathrm{D}}{\mathrm{v}} \frac{\partial \mathrm{C}(\mathrm{x}, \mathrm{t})}{\partial \mathrm{x}}\right|_{\mathrm{x}=\mathrm{o+}} \\ \lim _{\mathrm{x} \rightarrow+\infty} \mathrm{C}(\mathrm{x}, \mathrm{t})=0\end{array}\right\}$ para $\mathrm{t} \geq 0$

Aplicando transformada de Laplace a ambos lados de la EDP de la ecuación de contorno de la ecuación 10 tenemos:

$\mathcal{L}\left\{\frac{\partial \mathrm{C}}{\partial \mathrm{t}}\right\}=\mathcal{L}\left\{\mathrm{D} \frac{\partial^{2} \mathrm{C}}{\partial \mathrm{x}^{2}}-\mathrm{v} \frac{\partial \mathrm{C}}{\partial \mathrm{x}}\right\}$

Aplicando las propiedades se obtiene:

$$
\mathrm{s} \overline{\mathrm{C}}-\mathrm{C}(\mathrm{x}, 0)=\mathrm{D} \frac{\partial^{2} \overline{\mathrm{C}}}{\partial \mathrm{x}^{2}}-\mathrm{v} \frac{\partial \overline{\mathrm{C}}}{\partial \mathrm{x}}, \text { donde: } \mathcal{L}\{\mathrm{C}(\mathrm{x}, \mathrm{t})\}=\overline{\mathrm{C}}(\mathrm{x}, \mathrm{s})
$$

Sustituyendo la condición inicial, $C(x, 0)=0$, y reordenando términos se obtiene:

$\mathrm{D} \frac{\partial^{2} \overline{\mathrm{C}}}{\partial \mathrm{x}^{2}}-v \frac{\partial \overline{\mathrm{C}}}{\partial \mathrm{x}}-s \overline{\mathrm{C}}=0$

Aplicando transformada de Laplace sobre la primera condición de borde se obtiene:

$$
\mathcal{L}\left\{\mathrm{C}(0, \mathrm{t})-\left.\frac{\mathrm{D}}{\mathrm{v}} \frac{\partial \mathrm{C}(\mathrm{x}, \mathrm{t})}{\partial \mathrm{x}}\right|_{\mathrm{x}=0^{+}}\right\}=\mathcal{L}\left\{\frac{\mathrm{M}}{\mathrm{Q}} \delta(\mathrm{t})\right\}
$$

Como M y $Q$ son constantes y $\mathcal{L}\{\delta(\mathrm{t})\}=1$, la condición transformada queda como:

$\overline{\mathrm{C}}(0, s)-\frac{D}{v} \frac{\partial \bar{C}(0, s)}{\partial x}=\frac{\mathrm{M}}{\mathrm{Q}}$

Al aplicar transformada de Laplace a la segunda condición de borde: $\lim _{x \rightarrow+\infty} \bar{C}(x, s)=0$

Considerando a s como si fuera un parámetro "temporalmente", la ecuación diferencial parcial 11 se puede reescribir como una ecuación diferencial ordinaria (EDO). Las condiciones (12) y (13) también se pueden reescribir con respecto a x. Esto permite plantear el problema de contorno con EDO dado por (14). 
EDO: $\mathrm{D} \frac{\mathrm{d}^{2} \overline{\mathrm{C}}}{\mathrm{dx}^{2}}-v \frac{\mathrm{d} \overline{\mathrm{C}}}{\mathrm{dx}}-\mathrm{s} \overline{\mathrm{C}}=0$

$\bar{C}(0)-\frac{D}{v} \bar{C}^{\prime}(0)=\frac{M}{Q}$, Condiciones de borde: $\left\{\begin{array}{c}\bar{C}(0)-\frac{D}{v} \bar{C}^{\prime}(0)=\frac{M}{Q} \\ \lim _{x \rightarrow+\infty} \bar{C}(x)=0\end{array}\right\}$

La ecuación característica asociada a la EDO del problema 14 es: $\mathrm{Dm}^{2}-\mathrm{vm}-\mathrm{s}=0$,

Al resolver la ecuación característica, las condiciones de borde en forma similar a lo mostrado para el caso anterior, la solución del problema de borde con EDO 14 es:

$$
\bar{C}(x)=\frac{2 v M}{Q\left(v+\sqrt{v^{2}+4 D s}\right)} e^{\left(\frac{v-\sqrt{v^{2}+4 D s}}{2 D}\right) x}
$$

Dejando de considerar s como parámetro:

$\bar{C}(x, s)=\frac{2 v M}{Q\left(v+\sqrt{v^{2}+4 D s}\right)} e^{\left(\frac{v-\sqrt{v^{2}+4 D s}}{2 D}\right) x}$

Aplicando transformada inversa de Laplace y teniendo en cuenta la linealidad de la transformada se obtiene:

$C(x, t)=\frac{v M}{Q}\left(\frac{e^{-\frac{\left(x^{2}-2 v x t+v^{2} t^{2}\right)}{4 D t}}}{\sqrt{\pi D t}}-\frac{v}{2 D} e^{\frac{v x}{D}} \operatorname{erfc}\left(\frac{v t+x}{2 \sqrt{D t}}\right)\right)$

Factorizando el numerador del exponente del primer término, se obtiene:

$C(x, t)=\frac{v M}{Q}\left(\frac{2}{\sqrt{4 \pi D t}} e^{-\frac{(x-v t)^{2}}{4 D t}}-\frac{v}{2 D} e^{\frac{v x}{D}} \operatorname{erfc}\left(\frac{v t+x}{\sqrt{4 D t}}\right)\right)$

La ecuación 17 es igual a la citada por Kreft y Zuber, 1978 para esta condición de borde y coincide con la de recipiente cerrado (alteración de flujo debido a la toma de muestra del trazador a su paso por el reactor, donde se afirmó que no existía una solución analítica (Levenspiel, 1999).

\section{RESULTADOS Y DISCUSIÓN}

Inoculación y funcionamiento del reactor. Después de inoculado el reactor se dejó por carga durante 7 días alcanzando una eficiencia de remoción del $70 \%$ en términos de la DQO al sexto día. La temperatura ambiente promedio fue de $29,63 \pm 2,22{ }^{\circ} \mathrm{C}$ siendo inferior a las del afluente $\left(33,93 \pm 1,39{ }^{\circ} \mathrm{C}\right)$ y efluente $\left(31,62 \pm 1,83^{\circ} \mathrm{C}\right)$. El pH del afluente $(7,19 \pm 0,19$ y $7,26 \pm 0,18)$ fue ligeramente superior al efluente $(6,92 \pm 0,13$ y $6,90 \pm 0,18)$, en ambos casos permaneció cerca del rango de la neutralidad, el cual es recomendado para un buen desarrollo de la digestión anaerobia (Satoto, 2009). La alcalinidad del sistema fue de 236 $\pm 33,64$ $\mathrm{mgCaCO} / \mathrm{L}$, suficiente para mantener la estabilidad en el $\mathrm{pH}$.

La eficiencia de remoción de la carga orgánica, en términos de DQO, permaneció casi constante (cercana al $30 \%$ ) cuando el reactor funcionó con 3 entradas, y se incrementó hasta un máximo de $62,49 \%$ y $62,22 \%$ para la DQO total y DQO soluble respectivamente, cuando se modificó el número de entradas. La producción de biogás varió desde 0,165 a 0,456 $\mathrm{m}^{3}$ biogás $/ \mathrm{kgDQO}$, con un promedio de 0,280 $\mathrm{m}^{3}$ biogás/kgDQO, resultado similar a los obtenidos a escala real (Peña et al., 2006) de 0,341 a 0,434 $\mathrm{m}^{3}$ biogás/kgDQO removida. El porcentaje de metano varió entre $49,4 \%$ y $88,92 \%$ con un promedio de 72,32\%; estos resultados son similares a los encontrados a escala de laboratorio (Bermúdez et al., 2003). 


\section{Ajuste de los datos experimentales a los modelos de dispersión tradicionales}

Para determinar las bondades de ajustes de los modelos tradicionales y propuesto, se determinó la desviación media absoluta (DMA\%), desviación absoluta máxima que se indica en paréntesis, y la desviación media relativa (DMR\%) como medidas del error entre los datos calculados y experimentales (Valderrama y Alvarez, 2005), así como también la sumatoria de los errores al cuadrado (SSE) y raíz del error cuadrático medio (RMSE) usada como bondad de ajuste en la DTR (Zeng et al., 2005; Abou y Azizi, 2015). En la Tabla 1 se presentan las bondades de ajustes de los modelos tradicionales para cada uno de los tiempos de retención y condiciones estudiadas. Como es normal, la curva de DTR presentó valores de 0,0 al inicio y final, en estos casos no es posible determinar la desviación de los errores.

Como se observa, no existe un buen ajuste a los datos experimentales, los errores más bajos se obtuvieron con el modelo de TS, pero como se puede observar la DMA y DMR son elevados. Se resalta la importancia de la estimación de los errores ya que la SSE y la RMSE no dan una clara evidencia del verdadero ajuste. Se observó la limitación del modelo de TS cuando N resulta menor o igual a 1 (Martin, 2000) para el caso de to de 8 horas para la zona intermedia del reactor. El modelo TS sólo contempla como parámetro el valor de $\mathrm{N}$, lo que hace necesario calcular el valor del número de dispersión a través de la varianza para condición de recipiente cerrado (Lara et al., 2000; Gómez 2006; Sánchez y Cardona, 2009) o con el modelo GRra (Arroyave et al., 2005), que no obedecen al modelo de mayor ajuste ni a las condiciones de borde definidas en los ensayos hidráulicos. La literatura reporta resultados contradictorios con dispersión menor para el modelo de GDra que el de PD, (De Carlvalho, 2006) o se reporta el número de dispersión asumiendo condiciones de borde para recipiente cerrado y los datos se ajustan mejor al modelo de TS (Peña et al., 2006), sin garantizar las condiciones de borde de acuerdo a la inyección y recolección del trazador

También se han obtenido buenos ajuste de los datos de DTR con el modelo de Gauss pero el número de dispersión se hace a través de la varianza (Abou y Azizi, 2015), por otro lado el modelo de TS ha presentado buenos ajustes de manera parcial (Chen et al., 2015), esto se debe a la cola alargada que describe la curva de DTR. Si no se consideran las condiciones de borde adecuadas podemos llegar a resultados diferentes tal como ocurrió en un reactor a escala de laboratorio cuando se tomaron condiciones de GDra dadas por Levenspiel, 1999 (Lou et al., 2006), obteniendo mayores valores de dispersión al definir condiciones de borde adecuadas (Zeng et al., 2005), del mismo modo que se observó en un estudio a escala real (Avella, 2001; Peña et al., 2006), lo anterior indica que si no encontramos la dispersión del flujo como parámetro de ajuste del modelo podemos llegar a resultados y conclusiones diferentes, es por ello que esta investigación tiene un gran aporte al estudio del tipo de flujo presente en un reactor a través de un modelo de dispersión axial en un sistema de tratamiento de agua residual en reactores de flujo continuo.

\section{Ajuste de los datos experimentales al modelo de dispersión propuesto}

Comparando los resultados de la Tabla 1 con la Tabla 2, se pudo observar que los mejores ajustes se lograron con el modelo propuesto para las dos condiciones de borde. Para la inyección y recolección en flujo (IRF) se obtuvieron $21,5 \%<\mathrm{DMA}<32,7 \%$ y $2,2 \%<\mathrm{DMR}<-18,0 \%$, menores errores fueron encontrado para la inyección y recolección en el fluido residente (IRFR) con 5,0\%<DMA $<17,9 \%$ y $-1,7 \%<\mathrm{DMR}<2,7 \%$, los altos valores de la desviación absoluta máxima obedecen al comportamiento gaussiano de la curva de distribución de los tiempos de residencia del trazador que al tener valores cercanos a cero $(0,0)$ al inicio y al final, hace que un valor pequeño representa un error alto. El valor negativo de la DMR significa que el modelo subestimó los datos experimentales. A nivel general se lograron $0,0335<\mathrm{SSE}<1,7701$ y $0,0198<R M S E<0,1609$, e incluso la RMSE fue menor a los encontrados en un estudio de dispersión axial $(0,037<R M S E<0,175)$ realizado a escala de laboratorio (Zeng et al., 2005) Los pequeños valores de SSE y RMSE indican un buen ajuste como se muestra en la Tabla 2, con un alto porcentaje de recuperación del trazado, lo que brinda confianza en los resultados. El modelo IRF fue utilizados para evaluar el comportamiento hidráulico en un reactor anaerobio de doble cámara (RADCA) obteniendo buenos resultados (Rincón et al., 2010). Para observar de forma visual los ajustes de los datos experimentales a los diferentes modelos, se presenta una de las 10 corridas realizadas, que corresponde a la curva DTR a un to de 8 horas (Ver figura 2), evidenciándose un mejor ajuste con la simulación del modelo propuesto (IRF).

El número de dispersión obtenido en el efluente (comportamiento general del reactor) fue $0,2911<\mathrm{d}<0,5193$ cuando el reactor funcionó con 3 entradas, valores ligeramente mayores fueron obtenidos cuando se realizaron las modificaciones en las entradas $(0,3369<\mathrm{d}<0,7285)$, el incremento se debió a la mejora de la distribución del afluente. Revisando los valores de dispersión obtenidos en varios estudios, se pudo observar que la dispersión del flujo es menor para configuraciones cilíndricas con diámetros delgados ya que se impone un régimen de flujo pistón, pero los reactores a escala real conservan por lo general una forma rectangular o cuadrada, como fue el caso de esta investigación, de esta manera encontramos valores a escala de laboratorio en reactores cilíndricos con número de dispersión (d) menores a $0,078<\mathrm{d}<0,2115$ (Zeng et al., 2005, Ren et al., 2008) y aún menores en columnas tubulares con d< 0,05 (Abou y Azizi, 2015) y d $<0,0052$ (Bittante et al., 2014). 


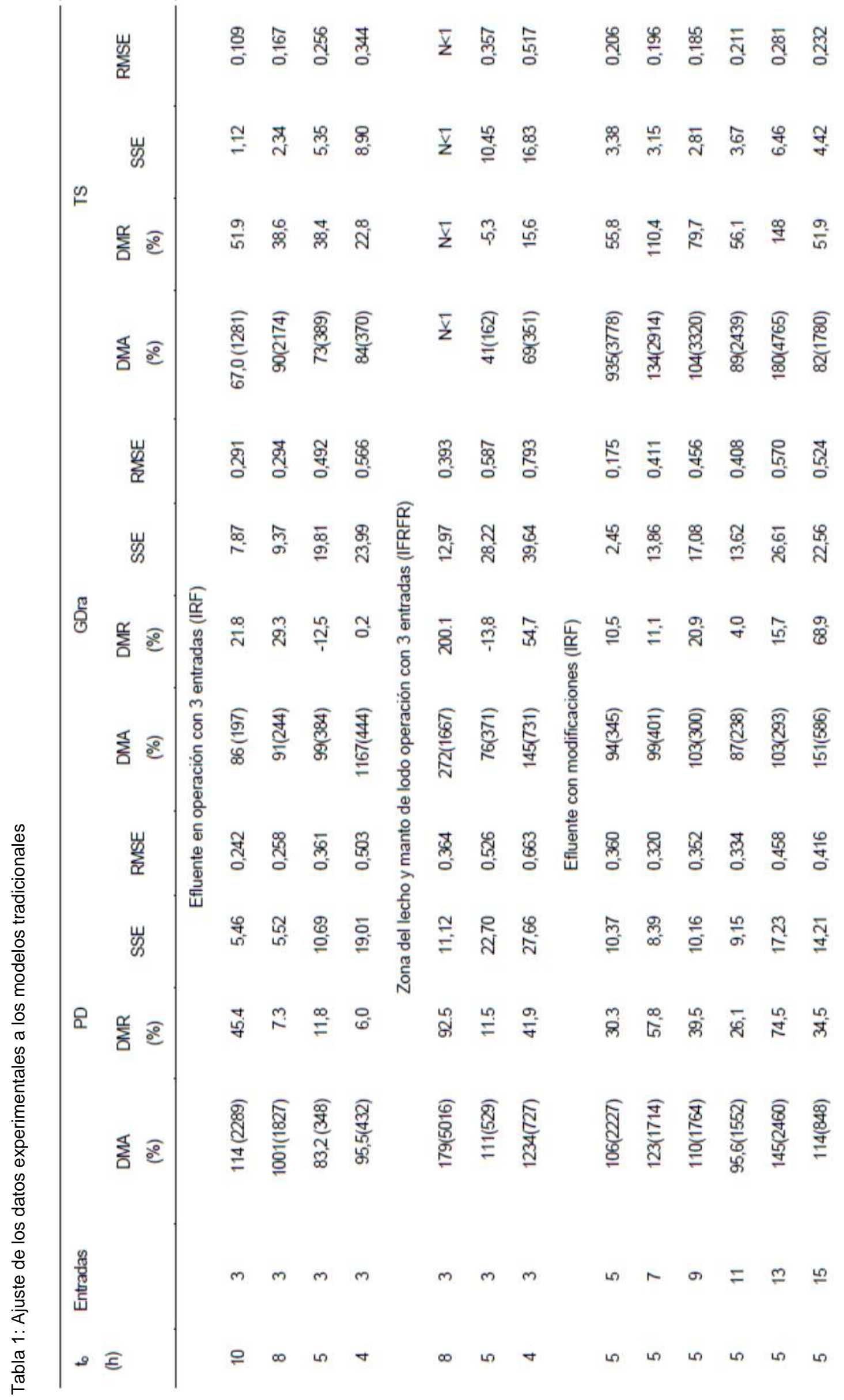


Tabla 2: Dispersión y ajuste de los datos experimentales al modelo propuesto

\begin{tabular}{|c|c|c|c|c|c|c|c|c|}
\hline$t_{0}(h)$ & Entradas & $\%$ & d & $\mathrm{D}\left(\mathrm{m}^{2} / \mathrm{h}\right)$ & $\mathrm{DMA}(\%)$ & $\operatorname{DMR}(\%)$ & SSE & RMSE \\
\hline \multicolumn{9}{|c|}{ Efluente en operación con 3 entradas (IRF) } \\
\hline 10 & 3 & 98,7 & 0,2911 & 8,662E-03 & $21,5(100)$ & $-18,0$ & 0,0345 & 0,0198 \\
\hline 8 & 3 & 101,7 & 0,3828 & 1,379E-02 & $24.6(99.4)$ & $-17,2$ & 0,0645 & 0,0279 \\
\hline 5 & 3 & 92,3 & 0,4209 & $2,439 E-02$ & $25,0(100)$ & $-13,0$ & 1,2090 & 0,1229 \\
\hline 4 & 3 & 95,4 & 0,5193 & 4,540E-02 & $32,7(147)$ & 5,2 & 1,1940 & 0,1279 \\
\hline \multicolumn{9}{|c|}{ Zona del lecho y manto de lodo operación con 3 entradas (IFRFR) } \\
\hline 8 & 3 & 98,9 & 1,3736 & $4,177 \mathrm{E}-02$ & $11.6(64,3)$ & -2.5 & 0,3614 & 0,0668 \\
\hline 5 & 3 & 97,4 & 1,3711 & $1,010 \mathrm{E}-01$ & $17,9(100)$ & -1.7 & 1,6342 & 0,1609 \\
\hline 4 & 3 & 87,7 & 1,3879 & 1,741E-01 & $5,0(41,9)$ & 2.7 & 0,4061 & 0,0797 \\
\hline \multicolumn{9}{|c|}{ Efluente con modificaciones (IRF) } \\
\hline 5 & 5 & 100,7 & 0,3369 & 1,952E-02 & $26,2(87,7)$ & $-3,8$ & 0,4338 & 0,0741 \\
\hline 5 & 7 & 93,0 & 0,4429 & 2,537E-02 & $27,2(143)$ & $-5,6$ & 0,2634 & 0,0570 \\
\hline 5 & 9 & 91,1 & 0,4211 & 2,598E-02 & $22,9(100)$ & $-7,9$ & 0,6521 & 0,0903 \\
\hline 5 & 11 & 98,1 & 0,4431 & 2,995E-02 & $26,8(99)$ & 4,3 & 0,2826 & 0,0598 \\
\hline 5 & 13 & 96,8 & 0,7285 & 4,049E-02 & $26,0(177)$ & 2,2 & 1,2800 & 0,1265 \\
\hline 5 & 15 & 91,8 & 0,6680 & 4,599E-02 & $28,5(100)$ & $-15,0$ & 1,0740 & 0,1166 \\
\hline
\end{tabular}

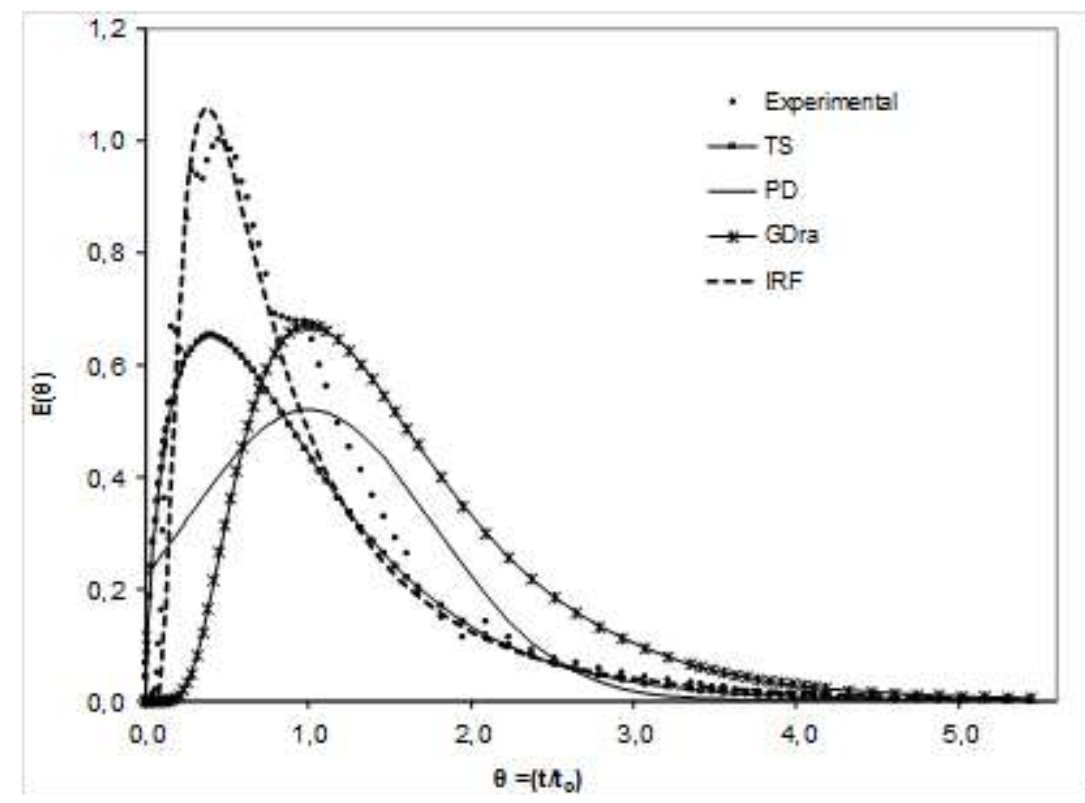

Fig. 2: Ajuste de los datos experimentales a los modelos de dispersión a to de 8 horas.

Como se puede observar en la Tabla 2, la dispersión obtenida en la zona de lecho y manto de lodo (parte inferior del reactor) fue mayor que en el efluente, registrando valores de $1,3711<\mathrm{d}<1,3879$, lo que es de esperarse por la distribución uniforme del flujo en el fondo del reactor y además en esta zona ocurre la degradación de la materia orgánica generándose una turbulencia por la producción de biogás. tal como se reportó en un lecho fluidizado (Kim y Kim, 1983), en un RAFA a escala real (Peña et al., 2006) y a escala de laboratorio (Morgan et al., 1997). Por lo anterior se puede concluir que en la zona del lecho y manto de lodo, el reactor describió un comportamiento de mezcla completa y a nivel general registró flujo disperso .al igual que varias investigaciones (Heertjes y Van der Meer, 1978; Bolle, 1986; Avella, 2001; Zeng et al., 2005; Ren et al., 2008). Cuando se evalúa el patrón de flujo se determinan sus fracciones: pistón, mezclada y zonas muertas (Ren et al., 2008), el cual fue determinado en esta investigación, pero no es objeto de ésta publicación.

De manera semejante a otros estudios (Avella, 2001; Zeng et al., 2005; Lou et al., 2006; Ren et al., 2008 y $\mathrm{Ji}$ et al., 2014), la dispersión del flujo es proporcional a la velocidad ascensional (diminución del tiempo de retención), lo que se debe al incrementos en el grado de coalición de las partículas suspendidas y el lodo que genera mayor producción de biogás (Ren et al., 2008), pero no se debe llegar a velocidades muy alta porque podría aumentar la fuerza de corte hidráulico disminuyendo la sedimentación de las partículas y consecuentemente deteriora la eficiencia de remoción (Mahmoud et al., 2003). 


\section{CONCLUSIONES}

De los argumentos presentados en los antecedentes, la discusión de los resultados expuestos en el artículo, se obtienen las siguientes conclusiones principales:

i) En todos los casos, el número de dispersión (d) obtenido es superior a 0,01, razón por la cual se puede afirmar que no ocurrió pequeña dispersión, pues el mezclado originado por la velocidad de entrada y el biogás generaron un buen mezclado en el reactor.

ii) El modelo propuesto para la inyección y recolección en flujo (IRF) e inyección en flujo y recolección en el fluido residente (IFRFR), presentó un buen ajuste a los datos experimentales de concentración del trazador, contrario a lo ocurrido con los modelo tradicionalmente usado.

iii) Existe una solución analítica para la condición de borde denominada recipiente cerrado que equivale al modelo IFRFR

iv) El coeficiente de dispersión se incrementó de forma proporcional con la velocidad ascensional tanto en la zona del lecho y manto de lodo como en el efluente.

\section{AGRADECIMIENTOS}

A la Universidad La Guajira; al Departamento de Ingeniería Sanitaria y Ambiental (DISA) por el apoyo financiero, al Centro de Investigaciones del Agua (CIA) de la Universidad del Zulia por su apoyo logístico. Al profesor Jaime Castillo de la Universidad de La Guajira, Colombia por su asesoría en matemáticas.

\section{REFERENCIAS}

Abou, K., y Azizi, F., Hydrodynamics and residence time distribution of liquid flow in tubular reactors equipped with screen-type static mixers, doi: 10.1016/j.cej.2015.05.100, Chemical Engineering Journal, (por suscripción), 279, 948-963 (2015)

APHA, AWWA y WPCF, Standard Methods for the Examination of Water and Wastewater, 20th edition U.S.A, ed. American Public Health Association. Washington D.C, USA (2005)

Arroyave, D., M. González, y D. Gallego, Evaluación del comportamiento hidráulico de un reactor UASB utilizado para el tratamiento de aguas residuales, VIII congreso Peruano de ingeniería química, 1-10, Lima, Perú, 24 al 27 de abril (2005)

Avella, G., Evaluación del comportamiento hidrodinámico de un reactor UASB y su influencia en la remoción de materia orgánica. Tesis de magíster, Facultad de ingeniería, Universidad del Valle, Cali, Colombia, P. 196, (2001)

Bermúdez, R.C., S. Rodríguez, M. Martínez, I. Adis y T. Brown, Ventajas del empleo de reactores UASB en el tratamiento de residuales líquidos para la obtención de biogás, Tecnología Química XXIII (2), 37-44 (2003)

Bittante, A., J. García-Serna., P. Biasi., F. Sobrón., y T.O. Salmi., Residence time and axial dispersion of liquids in Trickle Bed Reactors at laboratory scale, doi: 10.1016/j.cej.2014.03.062, Chemical Engineering Journal, 250, 99-111 (2014)

Bolle, W., J. Van Breugel., G., N. Kossen., W. Van Gils., An integrated dynamic model for the UASB reactor. doi: 10.1002 / bit.260281106, Biotechnology and Bioengineering, 28, 1621-1636 (1986)

Centro Panamericano de Ingeniería Sanitaria y Ambiental - CEPIS, Tratamiento de agua para consumo humano, Filtración Rápida, Evaluación de plantas de tecnología apropiada, Perú, 283 (2004)

Chen, Y., J. He, Y. Mu., Y-Ch. Huo., Z. Zhang., T. A. Kotsopoulos., y R. J. Zeng. Mathematical modeling of upflow anaerobic sludge blanket (UASB) reactors: Simultaneous accounting for hydrodynamics and biodynamics, doi: 10.1016/j.ces.2015.07.016, Chemical Engineering Science, 137, 677-684 (2015)

De Carvalho, K.Q., M. Salgado, F.H. Passig, y E.C. Pires, Resposta dinâmica de reator UASB submetido a cargas orgânicas e hidráulicas cíclicas: resultados experimentais, Chile. IX taller y simposio latinoamericano de digestión anaerobia, 513-519, Chile, 1-23 de octubre (2008) 
De Carvalho, K.Q., Reposta dinâmica de reator UASB em escala piloto submetido a cargas orgânicas e hidráulicas cíclicas: modelos matemáticos e resultados experimentais, Tesis doctoral, Universiad de São Paulo, Brasil (2006)

Devore, J. Probabilidad y estadística para ingeniería y ciencias, Quinta edición, 519, Editorial Thomson, D.F, México, (2001)

Elgeti, K., A new equation for correlating a pipe flow a reactor with a cascade of mixed reactors, doi: 10.1016 / S0009-2509, Chemical engineering Science, 51 (23), 5077-5080 (1996)

Gómez, M.G., Measurement and correlation of axial dispersion coefficient in a bubble column with a nonnewtonian liquid phase, Tesis doctoral. University of Puerto Rico, Mayagüez Campus, Puerto Rico (2006)

Gupta, R y A. Bansal, Effect of bed configuration on dispersion in a packed bed reactor, doi: 10.1021 / ie901376s, Ind. Eng. Chem. 49 (19), 9525-9528 (2010)

Heertjes, P., Van der Meer., R. Dynamics of Liquid Flow in an Up-Flow Reactor Used for Anaerobic Treatment of Wastewater, doi: 10.1002 / bit.260201007, Biotechnology and Bioengineering, (por suscripción), 20 (10), 1577- 1594 (1978)

Holley, E.R., Unified view of diffusion an dispersion. Journal of the Hydraulics Division Proceedings of the American Society of Civil Engineers, 2, 620-631 (1969)

Ji, Y.X., B. S. Xing., G. F. Yang., W.M. Ni., L. X. Guo., y R.C. Jin., Performance and hydrodynamic features of a staged up-flow ANAMMOX sludge bed (SUASB) reactor, doi: 10.1016/j.cej.2014.05.048, Chemical Engineering Journal, (en linea), 253, 298-304 (2014)

Kim, S.D., y C.H. Kim, Axial dispersion characteristics of three phase fluidized beds, doi: 10.1252/ jcej.16.172, Journal of chemical engineering of Japan, 16 (3), 172-177 (1983)

Kreft, A y A. Zuber., On the Physical meaning of the dispersion equation and its solutions for different initial and boundary conditions, doi: 10.1016 / 0.009 hasta 2.509 (78) 85.196-3, Chemical Engineering Science, (por suscripción), 33 (11), 1471-1480 (1978)

Lara, C.R., J. Díaz, y A. Bona, Evaluación hidráulica de un reactor UASB de compartimientos. Seminario Internacional: Tratamiento y Postratamiento de Aguas Residuales, 255-273, Universidad de Boyacá, Colombia, 22 a 25 de noviembre (2000)

Levenspiel, O., Chemical reaction engineering, $1^{\text {ra }}$ edición, 285, John Wiley and Sons, New York, USA (1972)

Levenspiel, O y W.K. Smit., Notes on the diffusion-type model for the longitudinal mixing of fluids in flow, doi: 10.1016/0009-2509(57)85021-0, Chemical engineering Science, 6, 227-233 (1957)

Levenspiel, O., Chemical reaction engineering, $2^{\text {da }}$ edición, 253-315, Ed. S.A. Wiley. New York, USA (1999)

Lou, S., B. Tartakovsk., Y. Zeng., P. Wu., S. Guiot., Fluorescence-based monitoring of tracer and substrate distribution in an UASB reactor, doi:10.1016/j.chemosphere.2006.03.045, Chemosphere, (en linea), 65, $1212-1220$ (2006)

Mahmoud N., G. Zeeman., H. Gijzen., G. Lettinga., Solids removal in upflow anaerobic reactors, a review, doi: 10.1016 / S0960-8524 (03) 00095-6, Tecnología Bioresource, (en linea), 90, 1-9, (2003)

Martin, A.D., Interpretation of residence time distribution data, doi: 10.1016 / S0009-2509 (00) 00108-1, Chemical Engineering Science, 55, 5907-5917 (2000)

Morgan, J.M., B. Jimenez, y A. Noyola, Tracer studies in laboratory and pilot scale UASB reactor, Envoiromental Technology, 18 (8), 817-825 (1997)

Passarini, K.C., F.M. Gamarra, R.M. Vanalle y J.C. Santana, Reutilización de las Aguas Residuales en la Irrigación de Plantas y en la Recuperación de los Suelos, doi:10.4067/S0718-07642012000100007 Inf. Tecnol (en linea), 23(1), 57-64 (2012) 
Peña M.R., D.D. Mara y G.P. Avella, Dispersion and treatment performance analysis of an UASB reactor under different hydraulic loading rates, doi: 10.1016 / j.watres.2005.11.021, Water Research, 40 (3), 445 $452(2006)$

Pérez, J.I., G.J. Aldana, M. Useche, N. Rincón, N. Bracho, y J. Mesa, Evaluación del modelo de dispersión axial de un reactor anaerobio de flujo ascendente (RAFA) a escala de laboratorio, http://goo.gl/HA2weJ, ISSN: 0254-0770, Revista Técnica de la Facultad de Ingeniería de la Universidad de Zulia 33 (3), 213 - 222 (2010)

Pérez, J.I., G.J Aldana. Modelación física de un reactor anaerobio de flujo ascendente (RAFA). http://goo.gl/jYrwGQ, ISSN: 0254-0770 (en línea), Revista Técnica de la Facultad de Ingeniería de La Universidad de Zulia 36 (2), 153 - 163 (2013)

Polprasert, Ch y K.K. Bhattarai, Dispersion model for waste stabilization ponds, dx.doi.org/10.1061/(ASCE)0733-9372(1985)111:1(45)), Journal of environmental Engineering, (por suscripción), 111 (1), 45-59 (1985).

Ren, T., Y. Mua., H. Yu., H. Harada., Y. Li., Dispersion analysis of an acidogenic UASB reactor, doi: 10.1016 / j.cej.2007.11.028, Chemical Engineering Journal, 142 (2), P. 182-189, (2008).

Rincón, N.C., A.A. Galindo, J.I. Pérez. Evaluación del comportamiento hidráulico en un reactor anaerobio de doble cámara (RADCA). http://bit.ly/1iDkvzU, ISSN: 0120-6230 (en línea), Revista Facultad de Ingeniería Universidad de Antioquia 61, 53-63 (2011)

Rocha, M.A., H. Normando, y C. Onofre, Aplição de tracadores em filtros anaeróbicos para avaliação das características hidrodinâmicas. Associação Brasileira de Engenharia Sanitária e Ambiental - Região Rio Grande do Sul (ABES/RS). XXVII Congresso Interamericano de Engenharia Sanitária e Ambiental, Porto Alegre, Brasil (2000)

Sánchez, J., S. Cardona., Evaluación del comportamiento hidráulico de un reactor aerobio y un reactor anaerobio, en una planta de aguas residuales domésticas a pequeña escala, ISSN 0121-5701 (en línea), Avances en Recursos Hidráulicos, 20, 65-80 (2009). http://www.bdigital.unal.edu.co/4767/, Acceso: 12 de Junio (2014)

Satoto, E.N., Anaerobic digestion of organic solid waste for energy production, Tesis doctoral. Universitat Fridericiana zu Karlsruhe (TH). Indonesien, 148 (2009)

Wehner, J.F. y R.H. Wilhelm, Boundary condiction of flow reactor, doi:10.1016/0009-2509(56)80014-6, Chemical Engineering Science, 6, 89-93 (1956).

Valderrama, J.O y Alvarez, V.H., Correct Way of Reporting Results when Modelling Supercritical Phase Equilibria using Equations of State, doi: 10.1002/cjce.5450830323, The Canadian Journal of Chemical Engineering, 83 (3), 578-581 (2005)

Wen C. Y. and Fan L. T. Models for flow systems and chemical reactors. Vol. 3, pp 581., Marcel Dekker, Inc., New York (1975)

Werner, A., H. Hotzl, P. Maloszewski, W. Kâss., Interpretation of tracer tests in karst systems with unsteady flow conditions, Karst Hydrology (Proceedings of Workshop W2 held at Rabat, Morocco, April-May 1997), 247, 15-26 (1997)

Zeng, Y., Mu, S., Lou, S., Tartakovsky, B., Guiot, S., Wu, P., Hydraulic modeling and axial dispersion analysis of UASB reactor, doi: 10.1016 / j.bej.2005.04.024, Biochemical Engineering Journal, 25 (2), 113$123(2005)$ 